\title{
Endogenous Archeological Sciences: Anatomy, Physiology, Neuroscience, Biochemistry, Immunology, Pharmacology, Oncology, Genetics as Instrument for A New Field of Investigation? Modern Global Aspects for A New Discipline
}

Luisetto $\mathrm{M}^{1 *}$, Naseer Almukhtar ${ }^{2}$, Ghulam Rasool Mashori ${ }^{3}$, Ahmed Yesvi Rafa ${ }^{4}$, Farhan Ahmad Khan ${ }^{5}$, Gamal Abdul Hamid ${ }^{6}$, Luca Cabianca ${ }^{7}$ and Behzad Nili-Ahmadabadi ${ }^{8}$

${ }^{1}$ Applied Pharmacologist, European specialist lab medicine, independent researcher, Italy

${ }^{2}$ Professor, University of Babylon, Iraq

${ }^{3}$ Professor of Pharmacology, People University of Medical \& Health Sciences for Women, Pakistan

${ }^{4}$ Founder and President, Yugen Research Organization, Independent Researcher, Bangladesh

${ }^{5}$ Associate Professor and Head Dept. of Pharmacology, Govt. Medical College, India

${ }^{6}$ Professor, Hematology Oncology, University of Aden, Yemen

${ }^{7}$ Luca Cabianca, Biomedical Labo, Italy

${ }^{8}$ PharmD, Nano Drug Delivery, USA

*Corresponding author: Luisetto M, Applied Pharmacologist, European specialist lab medicine, Independent Researcher, 29121, Italy.

Received Date: November 21, 2018

Published Date: November 27, 2018

\begin{abstract}
In this work is analyzed scientific literature involved in human evolution to be used as an archeological pathway to link different sciences in an overall new discipline. A rational classification of single evidence make possible to better understand under new light some physiological process. The archeological instrument to be applied in other field like biology or other sciences.
\end{abstract}

Keywords: Inside, Endogenous, Archeology, Physiology, New scientific discipline, Archeo-toxicology Archeo-oncology, Filogenetic, Ontogenetic, Pharmacological research

\section{Introduction}

Today is interesting to submit to the scientific world a new field of investigation more structured that We can denominate Endogenous archeology. A new field in which collect different research works from different discipline in order to correctly classify.

Endogenous process and structure of a sort of archeological provenience but today inside us. In example is possible to verify physio-pathology of limbic system (amygdala) related relevant facts involving human living also in today time.
Archeology science not to be consider only related human product and manufacts but also an inside disciple to verify archeological process related to mind- set kinetics and to other system or organs. Brain, mind, immunologic system and other relevant physiological functions are deeply influenced by a primitive structure and to deeply understand the meaning of this complex system inside us make possible to better explain today Human behavior and physiology and other process.

To better explain this approach is possible to think in example the protection factor involved in auto-prevent poisoning form toxic 
plants or maternal instinct to protect fetus and other as sexual behavior, sleeping profile, fear and anger management, cooperative behavior et other strictly related whit evolutive pathways.

\section{Other facts can be considered to adequately set the endogenous archeo- structure}

The emato- encefalic barrier can be related to a ancestral world with higher toxic risk then the actual environment?

The same instinct is related to ancestral process.

Why in human brain are present oppiaceus receptor strictly related to opiaceus alkaloids?

And why in some neurological disease are often present cellular inclusion like prot. TAU and similar?

Is possible verify that in hibernant mammalians reduction of metabolism make possible to adequate survive in real prohibitive condition.

And related Neurotransmitter and receptorial status: this can be related to ancestral needs?

(Sympatic system so named by GALENO (130-200 DC) to describe nerves functions as link with periferical organs. A synaptic coordination between various part of the body, Prasimpatic: near to sympatic system, and other receptorial system like dopaminergic, seroto ninergic, purinergic, oppiacei receptors and so on)

Why insulin human production follow a circadian profile related the food level of intake?

Why bowel contract itself like ancient warms?

And related Maslow's hierarchy of needs why in the basis of piramids are present physiological need?

And related atetosic - tentacular movement in HUNTINGTON COREA disease? Inaccurate movements and in coordinated and its similarity with some acquatic invertebrated (see nervous system of this last compared to human).

Other examples can be: the atrofized men breast, human coccige, peach fuzz in al body, and other vestigial organs related to evolutionism like Ciecal appendix, ancestral erbivor residual bowel, navel, timus ant its immunologic profile.

Vomero nasal organ, now out of encephalon, in past function in detect other individuals ferormons

Darwin lobuli: residual structure by which animals can moveand better orienting auricolar pavilions.

L-gulonolattone oxidase pseudo- gene: present in superior anuimals but in human not codify for no one gene. The human Eterodontic theet related the actual dietary behavior, not necessary a great functional differentiation between teeth (canins)

And Observing also the differents in bipedal and in quadrupedal primates; The monkeys show the same rate in urinary infections like actual humans?

They need the same amount of antimicrobials/year due by UTI infectious disease? and why?
Verifying the evolution and final status of the lower urinary tract position related to gravity is possibile to verify the best condition to have maximum urinary flow also in actual bipedal humans.

The human osmotic blood pressure related to NACL concentration remember an evolution from sea.

Other examples can be the Vomit protective reflex, and related genetic profile: Human and other organism Junk DNA not codifing.

Very interesting is also the Brain evolution: palleocortex (mantein the olfactory function), archicortex (and its affettivity controI in first mammalians), neocortex related also to cognitive process.

The Primitive brain (reptilian brain or spinal bulble) is involved in involuntary movement, heart rate, breath et other, the intermediate brain or limbic system involved in emotion like fear anger, sensitive and motor responce (first mammalian) and neocortex associative (associated cognitive and relational functions) show a $\mathrm{n}$ evolution of structure associate to a more complex functions.

The Unicellular or pluricellular and then in Vertebrates evolution in motor systems (in fish, reptile, birds, mammalians) show a common evolutive way.

Ancient Fish presented limited motory function related to the see environment (under a spinal cord control and pallidus nucleus) in an ondulatory movement involving full body.

Reptile and anfibius developed other motor abilities related the new environment out of sea: new and complex motor need in the shift form sea to paludes and ground the Development of Striatus nucleus in reptile (invoved in crawl movement), as well as an high development of cerebellum in birds, improved movement of the body extremities and not only of the full body like in fish and reptile. Other High sensorial organ in birds as instrument to facilitate flying.

Birds take care of progeny, lives in family groups (on contrary of reptile) present higher and new instict In evolution in mammalians increased the encefalic control and reduced the automatism.

See in example progression movement in child (6 month -1 year): oscillate like fish, slither like reptile, crawl like mammalian, climb up and the walk. In mammalians evolution $90 \%$ of neurocortex is involved in associative role ( $10 \%$ sensitive and motor).

In The gradual evolution of vertebrates with adding of new sovra structure in brain the more recent level not needed other conduction ways to connect central nervous systems to muscle.

In striatus polineuronic way present a slow process and so in neurocortex a new direct way named pyramidal (new system named pyramidal added to the old system named extrapitramidal).

Pyramidal system make possible more precision in movement and rapidity in muscel response.

In mammalians tele- encefalization reduced the spine cord control in relation to in birds (in this last more higher motor automatism). 
And in other animals is possible to see related evolutive process

In cetaceus: bone draft posterior limb.

Wings in not flying birds, Pelvic belt in snake.

Rudimental eyes in Amblyopsidae fish.

Notocord vertebrate evolution and metameric organization Gill evolution et other.

\section{Material and Methods}

with an observational approach some relevant literature (from PUBMED database) are analysed in order to produce a final conclusion and to produce an Researchgate PROJECT dedicated opened to many researcher from different field like: neuroscientist, biologist, archeologist, psychologist, genetics, immunologist anatomist, physiologist and many other.

\section{Results}

\section{From scientific literature is possible to observe in article}

Amygdala pharmacology and crime behavior, dysfunctions to be considered as a disease: "neuro-pharmacology research of amygdala can give the response also using imaging Techniques. Studies demonstrated that Amygdala activation is involved in aggressive behavior" [1].

\section{And in publication Mindset kinetics and some depression status}

A new quantitative model under biochemical - toxicology approach? 2018 is reported: "According Pubmed Health database: Depression is a state of low mood and aversion to activity that can affect a person's thoughts, behavior, feelings and sense of wellbeing (moderate or severe).

Can we consider some depression status due to high amount of stressant stimulus? (Or) continuous stress in a limited (or long) period? How can react mindset and brain in management an high amount of negative stressing thinking? Observing relevant literature also mindset kinetics must be considered to better classify this kind of disorder under a specific endogenous-exogenous biochemicaltoxicological aspect.

Concept as Kinetics, reaction velocity limits, saturation of the systems, residual buffer properties are currently used in biochemistry and related discipline.

This concepts can be applied also in some depression condition to better explain some biological phenomena? [2]

\section{And in article Brain and Transmission Signal Modulation 2017}

"In activation of amygdala the response is more rapid than when involved the frontal cortex "[3]

\section{Carey HV et al writed}

Mammalian hibernators undergo a remarkable phenotypic switch: it involves great changes in physiology, morphology, and behavior in response to periods of unfavorable environmental conditions. The ability to hibernate is found throughout the class Mammalia and appears to involve differential expression of genes common to all kind of mammals, rather than the induction of novel gene products unique to the hibernating state. The hibernation season is characterized by extended bouts of torpor, minimal body temperature $(\mathrm{Tb})$ can fall as low as -2.9 degrees $\mathrm{C}$ and metabolism can be reduced to $1 \%$ of euthermic rates. Many biochemical physiological processes exploit low temperatures to lower reaction rates but retain the ability to resume full activity upon rewarming. Other critical functions must continue at physiologically relevant levels during torpor and be precisely regulated even at $\mathrm{Tb}$ values near 0 degrees $\mathrm{C}$. Research using new instruments as molecular and cellular biology is beginning to reveal how hibernators survive repeated cycles of torpor and arousal during the hibernation season. Genomic and proteomic technologies are needed to further better define the differentially expressed genes that distinguish the summer euthermic from winter hibernating states. Detailed understanding of hibernation from the molecular to organismal levels should enable the translation of this information to the development of a variety of hypothermic - hypometabolic strategies to improve outcomes for human and animal health [4-6]."

\section{According Davidson I}

"archeology of cognition is concerned primarily with the evolutionary emergence of the cognition particular to modern humans but there is an implication for the evolution of cognition among modern humans. Archeological evidence can provide important insights into the evolutionary emergence of human cognition, but theoretical considerations are fundamental in understanding what sorts of cognition there might have been between the ape-like common ancestor and modern humans. Archeology is the only source of evidence for the behavior associated with such theoretical stages. Cognitive archeology, therefore, involves an iterative interaction between theory from outside archeology and more or less direct evidence from the past. This review considers the range of possible evidence from archeology and genetics and summarizes the results of analysis of non-human primates particularly to assess characteristics of the last common ancestor (LCA) of apes and humans. The history of changes in size and shape of the brain since separation from other apes introduces the need to assess the appropriate cognitive theories to interpret such evidence. The review concentrates on two such approaches: Baddeley's working memory model as interpreted by Coolidge and Wynn, and Barnard's interacting cognitive subsystems as it has been elaborated to define the cognitive conditions for hominins between the LCA and modern people. Most of the rest of the review considers how the evidence from stone tools might be consistent with such theoretical models of cognition. This evidence is consistent with views that modern human behavior only emerged in the last 100,000 years (or so) but it gives an explanation for that in terms of cognition [7]."

\section{Simon Neubauer et al showed that}

"Modern humans have large - globular brains that distinguish them from their extinct Homo relatives. The characteristic globularity develops during a prenatal / early postnatal period 
of rapid brain growth critical for neural wiring and cognitive development. However, it remains unknown when and how brain globularity evolved and how it relates to evolutionary brain size increase. On the basis of Tc scans and geometric morphometric analyses, we analyzed endocranial casts of Homo sapiens fossils $(\mathrm{N}=20)$ from different time periods. Our scientific data show that, about 300,000 years ago, brain size in early Homo. sapiens already fell within the range of present day humans. Brain shape, evolved gradually within the $H$. sapiens lineage, reaching present-day human variation between about 100,000 - 35,000 years ago. This process started after other key features of craniofacial morphology appeared modern and paralleled the emergence of behavioral modernity as seen from the archeological record. Our findings are consistent with an important genetic changes affecting early brain development within the $\mathrm{H}$. sapiens lineage since the origin of the species and before the transition to the Later Stone Age and Upper Paleolithic that mark a full behavioral modernity [8]. "

\section{Bonett RM}

"Analyzing variation in rates of evolution can provide an important insights into the factors that constrain trait evolution, as well as those that promote diversification. Metazoan endocrine systems exhibit an apparent variation in evolutionary rates of their constituent components at multiple levels, yet few studies have quantified these patterns and analyzed them in a phylogenetic context. This may be in part due to historical and current data limitations for many endocrine components and taxonomic groups. Recent technological -advancements such as high throughput sequencing technique provide the opportunity to collect largescale comparative data sets for even non-model species that will produce a fertile data landscape for evolutionary analyses of nucleic acid and amino acid based endocrine components. I summarize evolutionary rate analyses that can be applied to categorical and continuous endocrine traits, and also those for nucleic acid and protein-based components. I emphasize the analyses that could be used to test whether other variables (ecology, ontogenetic timing of expression.) are related to the patterns of rate variation and endocrine component diversification. The application of phylogenetic based rate analyses to the comparative endocrine data will greatly enhance our understanding of the factors that have shaped endocrine system evolution [9]." (9)

\section{Charles L. Nunn et al}

"Sleep is essential to cognitive function and health in humans, yet the ultimate reasons for sleep-i.e. 'why' sleep evolved-remain mysterious. We integrate the findings from human sleep studies, the ethnographic record, and the ecology and evolution of mammalian sleep to better understand sleep along the human lineage and in the modern world. Compared to the other primates, sleep in great apes has undergone substantial evolutionary change, with all great apes building a sleeping platform or 'nest'. Further evolutionary change characterizes human sleep, with humans having the shortest sleep duration, yet the highest proportion of rapid eye movement sleep among the primates. These changes likely reflect that our ancestors experienced fitness- benefits from being active for a greater part of the $24 \mathrm{~h}$ cycle than other primates, potentially related to advantages arising from learning, socializing and defending against predators and hostile conspecifics. Perspectives from the evolutionary medicine have implications for understanding sleep disorders; we consider these perspectives in the context of : insomnia, narcolepsy, seasonal affective- disorder, circadian rhythm disorders and sleep apnea. We also identify how the human sleep today differs from sleep through most of human evolution, the implications of these changes for global health and health disparities. Our review highlights importance of the phylogenetic comparisons in understanding human health, including well-known links between sleep, cognitive performance and health [10]".

\section{Müller M}

"Parasitic amitochondriate protists, representatives of early branches of the eukaryote evolution, differ considerably in their central, energy metabolism from mitochondrion-bearing cells. Differences are: significant metabolic functions of inorganic pyrophosphate, major role of iron-sulfur proteins in key metabolic steps and in hydrogenosome-bearing organisms the disposal of electrons by $\mathrm{H} 2$ formation. Cytochrome mediated electrontransportand electron transport-linked phosphorylation are absent. All the proteins which have been sequenced so far were found to be homologous to isofunctional proteins from other organisms. few reactions are catabolized by the proteins which are not homologous to enzymes performing similar reactions in other eukaryotes. Two significative different types of metabolism of amitochondriate protists can be distinguished: (1) without compartmentation and (2) with cytosol/hydrogenosome compartmentation. These metabolic- types have conserved certain traits present in ancestral eukaryotes before mitochondria became established [11]. “

\section{Marco Fondi, et al}

"It is assumed that primordial cells had small genomes with simple genes coding for enzymes able to react with a wide range of chemically related substrates, interconnecting different metabolic routes. New kind of genes coding for enzymes with a narrowed substrate specificity arose by paralogous duplications of ancestral ones and evolutionary divergence. So new metabolic pathways were built up by primordial cells. Useful hints to disclose the origin / evolution of ancestral metabolic routes and their interconnections can be obtained by comparing sequences of enzymes involved in the same or different metabolic routes.

The lysine, arginine, and leucine biosynthetic routes represent very interesting study-models. Some of the lys, arg, leu genes are paralogs; this led to the suggestion that their ancestor genes might interconnect the three pathways. The aim of this work was to trace the evolutionary pathway leading to the appearance of the extant biosynthetic routes and to try to disclose the interrelationships existing between them and other pathways in the early stages of the cellular evolution [12]"

\section{According Dariush D FARHUD et al}

"The evolution of human blood groups, without doubt, has a history as old as man himself. There are at least 3 hypotheses about the emergence and mutation of human blood groups. The Global distribution -pattern of blood groups depends on various 
environmental factors, such as disease, climate, altitude, humidity. In this study, the collection of main blood groups $\mathrm{ABO}$ and $\mathrm{Rh}$, along with some minor groups, are presented. Various investigations of blood groups from Iran, particularly a large sampling on 291857 individuals from Iran, including the main blood groups $\mathrm{ABO}$ and $\mathrm{Rh}$, as well as minor blood groups such as Duffy, Lutheran, Kell, KP, Kidd, and Xg, have been reviewed.

It was not until 1900, when Karl Landsteiner at the Vienna univ., discovered why some blood transfusions were successful while others could be deadly. Landsteiner discovered the ABO bloodgroup system by mixing the red cells and serum of each of his staff. He demonstrated that: the serum of some people agglutinated the red cells of others . From these first experiments, he identified three types, called A, B and C (C was later to be re-named 0 for the German "Ohne", meaning "without", or "Zero", "null” in English). The fourth less frequent blood group $\mathrm{AB}$ was discovered one year later., Landsteiner received the Nobel Prize in physiology and medicine for his work.

The gene that determines human ABO blood type is located on the chromosome 9 ( $9 \mathrm{q} 34.1)$ and is called ABO glycosyl-transferase. The ABO locus has three main allelic forms: $A, B$, and 0 , as mentioned above and each of them is responsible for production of its glycoprotein. The combination of alleles that are inherited from parents that determines which glycoproteins (antigens) are found on persons' blood cells and thereby their ABO blood type.

Genesis and Evolution: investigations have demonstrated on monkeys; human blood groups are very old genetic indicators which have evolved during a several million years. Based on the primary races hypothesis, it was thought that in the three major races of man, blood groups A in Europe, B in Asian, finally 0 in South America have been emerged and gradually due to the migration and mixing of the races, became the present situation. But we know that in each continent, the isolated populations are seen that have completely different blood groups. For example, there is relatively high prevalence of blood group 0 in Siberian inhabitants; also, this blood group is very common in some parts of Switzerland.

Based on this theory, the old races have 0 blood group, such as Red Indians of South America, and Eskimos that among them the frequency of 0 blood group is between $75-100 \%$. While in most of recent ethnic groups A and B blood groups are dominant.

In other hypothesis, the first blood group had been $\mathrm{AB}$ blood group, which gradually and over the time due to genetic mutations was resulted in A and B and finally $\mathrm{O}$ blood groups . Base on this theory, perhaps a few million years ago all people have had type 0 blood only, which is more resistant against many infectious diseases. After discovery of the first human blood groups (ABO) by Karl Landsteiner in 1901 , gradually from 1927, other blood groups were also discovered and reported which its collection is given in. It is important to mention that Landsteiner together with his American colleague Alexander Wiener discovered the Rh blood group and reported it in 1940, 1941. Karl Landsteiner was born on 14th June 1868, in Vienna, Austria; he died on 26th June 1943 AD, at 75 years old, in the United States.
Landsteiner in his 17th scientific paper in 1901 reported blood group $\mathrm{ABO}$ which was displayed at the beginning with the letters ABC. In 1930, he received the Nobel Prize in Medicine for his discovery. In addition to the known blood groups, nearly twenty public antigens and also sixty-specific antigen or family antigen (Private Antigens) have been reported.

Moreover, the main blood groups $\mathrm{ABO}$, gradually discovered and reported which the most notably of them are as follows:

- A subgroups, including A1, A2, A3, and also rare types A4, A5, A6, Z, X, End, boutu, g, i.

- $\quad$ B subgroups, including B1, B2, B3, and rare types $\mathrm{w}, \mathrm{x}, \mathrm{v}$, m.

- $\quad$ Subgroups, including 01, 02, 03, and other types such as Yy, Hh, Xx, and Bombay [13]."

\section{According de Boer B}

"Speech is the physical signal used to convey spoken language. Because of its physical nature, speech is both easier to compare with other species' behaviors and easier to study in the fossil record than other aspects of language. I argue that convergent fossil evidence indicates adaptations for complex vocalizations at least as early as the common ancestor of Neanderthals and modern humans. I argue that it is unlikely that language evolved separately from speech, but rather that gesture, speech, and song coevolved to provide both a multimodal communication system and a musical system. Coevolution must also have played a role by allowing both cognitive and anatomical adaptations to language and speech to evolve in parallel. Although such a coevolutionary scenario is complex, it is entirely plausible from a biological point of view [14]."

\section{Cecilia Heyes}

"Humans are animals that specialize in thinking and knowing, and extraordinary cognitive abilities have transformed every aspect of our lives. In contrast to our chimpanzee cousins and Stone Age ancestors, we are complex political, economic, scientific and artistic creatures, living in a vast range of habitats, many of which are our own creation. Research on human cognition evolution asks what types of thinking make us such peculiar animals, and how they have been generated by evolutionary processes. New research i looks deeper into the evolutionary history of human cognition and adopts a more multi-disciplinary approach than earlier Evolutionary Psychology. It is informed by comparisons between humans and a range of primate and non-primate species, and integrates findings from anthropology, archaeology, economics, evolutionary biology, neuro-science, philosophy and psychology. Using these methods, recent research reveals profound commonalities, as well striking differences, between human and non-human minds, and suggests that the evolution of human cognition has been much more gradual and incremental than previously assumed. It accords crucial roles to cultural evolution, techno-social co-evolution and gene culture co-evolution. These have produced domain-general developmental processes with extraordinary power that makes human cognition, and human lives, unique. 
Chimpanzees lead quite interesting lives. They build nests, form alliances with other members of their troop and use simple tools, sticks to fish for termites and stones to open nuts. we are reminded by comedic images of chimpanzees wearing clothes and using computers, the lives of our closest evolutionary relatives are very different from our own. Translated as 'wise man', Homo sapiens is an optimistic sobriquet; translated as knowing man it is merely descriptive of our species. We are animals that specialize in thinking and knowing-in cognition-and our extraordinary cognitive powers have enabled us to do remarkable things. We have transformed our eating habits with agriculture and cooking, and transformed our habitats with buildings, bridges and roads. Compared with our chimpanzee cousins, we can travel over vast distances, moving our whole bodies in cars, planes and space craft, and moving our minds to yet more remote places with radio telescopes and electron microscopes. We are political and economic animals, negotiating agreements that affect millions of people, and trading instantly in rarefied currencies with completely anonymous strangers in different time zones. We know about time, we understand it to some degree and we can measure it precisely. We communicate with symbols-spoken and written languages-and using these languages, we have developed extensive knowledge of our own history and diversity, and about all aspects of the natural and physical worlds. Our lives are enriched by a fabulous range of beautiful, intricate and provocative objects-by art, architecture, music and dance-and, in addition to developing the weaponry of 'shock and awe', we engage in sports, complex rituals that channel and redirect the impulse to fight.

How has evolution produced creatures with minds capable of these remarkable feats? They represent new thinking about new thinking; leading edge, evidence-based theory about the new forms of cognition that emerged in the course of human evolution. The new theory and evidence come from a range of disciplines, including anthropology, archaeology, economics, evolutionary biology, neuroscience, philosophy and psychology. The new forms of cognition include causal reasoning, imitation, language, metacognition and theory of mind.

Over the past 25 years, research on the evolution of human cognition has been dominated by a type of evolutionary psychology promoted most prominently by Cosmides and Tooby. This framework, which I will identify using initial capitals ('Evolutionary Psychology'). It suggests that the human mind consists of a large collection of computationally distinct 'modules'. Each of these modules is a way of thinking that was shaped by natural selection to solve a particular type of problem faced by our Stone Age ancestors-for example, communicating, prey stalking, disease avoidance, mate choice and coalition formation. Evolutionary Psychology's central metaphor is the Swiss Army knife. It casts the evolved human mind as a set of cognitive gadgets, each specialized to learn, remember and reason about particular types of information. It has drawn attention to the need to integrate cognitive science with evolutionary biology in order to explain not only how brains and behaviour have evolved, but also the evolution of the 'middle man'- the cognitive processes, often characterized as computational software, which are instantiated by the brain and control behaviour. The new thinking that runs through this theme issue, sees the human mind as more like a hand than a Swiss Army knife. The hand is a multi-purpose instrument of a very different kind to the Swiss Army knife. It has a deep evolutionary history, rooted in the earliest emergence of the pentadactyl limb, and incorporates many genetic adaptations. The human hand is also capable of performing a wide / open-ended variety of technical and social functions. It can strip the defensive spines from a piece of fruit, making it safe to eat, but in Thai dancing it can also signal the smallest nuances of emotion. The human hand performs with equal facility a vast array of tasks that natural selection did and did not 'foresee'.

This paper introduces the theme issue by contrasting our 'new thinking' with Evolutionary Psychology in relation to three closely related questions about the evolution of human cognition: When did the most important changes take place? How did the changes happened? What have the changes produced [15]?

According paper Evolutionary change in agriculture: the past, present and future in 2010 reported that:

We draw together a series of diverse studies that provide a sample of some of the ways in which evolution driven by both conscious and unconscious selection by humans has shaped the development of modern agriculture. Agriculture has been a crucible of evolutionary change ever since its inception thousands of years ago, and this change permeates agricultural endeavours at all levels of biological organisation, ranging from the individual gene through to whole communities. Agro-ecosystems thus provide one of the most cogent examples of situations where anthropogenic effects are major determinants of biotic interactions within and among species and communities, suggesting a central role for the application of evolutionary principles. This is particularly the case, given global concerns regarding food production/food security, and increasingly, the expectation that agricultural productivity gains must be achieved with greater efficiencies, and reduced environmental impact.

In the development of human society, the shift to a relatively settled way of life from a nomadic hunter-gatherer life-style was enormously profound and made possible only by the development and adoption of early agricultural practices. This change in human life-style had major impacts on the environment of agriculture and hence the traits and characteristics of plants and animals that were favoured both consciously and unconsciously by humans. Very often traits suited to species growing without human protection/ husbandry were not suitable for agriculture and were rapidly lost (seed shattering), while others were specifically favoured by the more protected environment agriculture provided, and increased in frequency (seed retention).

As agriculture developed, the environment of the field and paddock became increasingly differentiated from that of the natural environments in which plants and animals originally evolved. For plants, nutrient availability generally rose, plant density and genetic uniformity increased, and the balance in competition shifted from inter-specific considerations to intra-specific ones 
(particularly with the general reduction in species diversity). Tilling and crop rotation were further agronomic practices that were introduced partly for fertility reasons but also for control of natural enemies, as rotation causes shifts in whole fungal pathogen communities. Another generation of changes to tilling practices minimum tillage - is to some extent now altering ecological and evolutionary interactions at the plant-soil interface towards a different set of fungal control issues. For animals, domestication created a more predictable environment with increased resource availability during harsh times and protection from predators, but increased threats from contagious diseases, all subtly influencing the evolutionary make-up of our livestock.

In last few thousand years, domestication, selection and hybridisation, both unconscious and conscious, has also led to significant changes in the appearance of plants and animals and their nutritional value.

Examples are seen in virtually all plant and animal species that are farmed. In horticulture, this diversity is often highly prized in the form of different varieties that are preserved for subtle variations in flavour, texture or simply appearance (e.g. in potato, tomato, apple). In a similar way, extensive selection in farmyard fowls (chickens, ducks, geese and turkeys), and in pigs, sheep and cattle have given rise to very many distinctive breeds that differ in milk production, flesh texture and flavour, and obvious appearance, as well as in less obvious traits, such as patterns of social behaviour. Extensive agriculture has also seen similar major changes that have resulted in significant increases in yield and productivity. In plants one of the most dramatic changes to have occurred within a species is found in the emergence of modern high-yielding hybrid maize from its close relative teosinte, and the subsequent application of a number of induced mutations and the introduction of an F1 hybrid system. Similarly, dramatic changes have occurred in meat and fibre production and quality in selected beef and sheep varieties respectively. In the history of agriculture, changes in agronomic and animal husbandry practices and in the crops and animals being farmed have had collateral effects that have changed the balance and intensity of different selective forces. This has been particularly apparent in plants where increasing nutrient status (particularly the nitrogen) generally favours the growth and development of insect and fungal pests - the numbers of which are frequently then further exacerbated by increased plant density (this increases humidity and thus conditions for fungal spore germination; and reduces inoculum loss during transmission) and genetic uniformity of crops. As selection by humans shifted to a conscious understanding of genetics, the focus also increasingly centred on accumulating single major gene traits because these could be more readily manipulated [16]."

\section{F. Lucaet al writed that}

"Through the cultural innovation and changes in habitat and ecology, there have been a number of major dietary shifts in human evolution, including meat eating, cooking, and those associated with plant and animal domestication. The identification of signatures of adaptations to such dietary changes in the genome of extant primates humans included may shed light not only on the evolutionary history of our species, but also on the mechanisms that underlie common metabolic diseases in modern human populations. The evolutionary history of hominins has been characterized by significant dietary changes, which include the introduction of meat eating, cooking, and the changes associated with plant and animal domestication. Decades of anthropological research studies have been devoted to elucidating this dietary history, in part because these shifts were likely associated with major anatomical and cultural changes (e.g., the increase in relative brain size and the advent of modern civilization via agriculture). This reconstruction is crucial for understanding the evolutionary context of our modern diets and the diseases often associated with them.

In parallel with historical reconstruction of hominin diets, molecular evolutionary analyses have been used to interrogate the genome for signals of genetic adaptations to different dietary regimes. A major advantage of many evolutionary genetic approaches is that they do not necessarily require strong assumptions about the specific genes and alleles that were targets of diet-related selective pressures. Evolutionary genetic analyses have the potential not only to inform existing adaptive hypotheses of hominin dietary history, to help generate new ones.

We bring together these 2 areas of inquiry, namely anthropology and evolutionary genetics, to highlight their recent findings related to human dietary history and to discuss the limitations of different approaches. We start by providing a brief overview of the major dietary shifts in hominin evolution and discussing evolutionary genetics methods and approaches used to identify signals of natural selection. We then review the results of genetic studies aimed at detecting the loci that played a major role in dietary adaptations and conclude by outlining the potential of future research studies [17].

\section{Tattersall writed that}

"Human beings are distinguished from all other organisms by their symbolic way of processing information about the world. This unique cognitive style is qualitatively different from all the earlier hominid cognitive styles and is not simply an improved version of them. The hominid fossil/archaeological records show clearly that biological and technological innovations have typically been highly sporadic, and totally out of phase, since the invention of stone tools some 2.5 million years ago. They also confirm that this pattern applied in the arrival of modern cognition: the anatomically recognizable species Homo sapiens was well established long before any population of it began to show indications of behaving symbolically. This place the origin of symbolic thought in the realms of exaptation, whereby new structures come into existence before being recruited to new uses, and of emergence, whereby entire new levels of complexity are achieved through new combinations of attributes unremarkable in themselves. These phenomena involve entirely routine evolutionary -processes; special as we human beings may consider ourselves, there was nothing special about the way we came into existence. Modern human cognition is a very recent acquisition; and its emergence ushered in an entirely new pattern of technological (and behavioral) innovation, in which 
constant change results from the ceaseless exploration of the potential inherent in our new capacity [18]."

\section{According Bar-Rogovsky $\mathrm{H}$ et alshowed that}

"Serum paraoxonases (PONs) are detoxifying lactonases that were first identified in mammals. 3 mammalian families are known: PON1, 2, and 3 that reside primarily in the liver. They catalyze the same reaction, lactone hydrolysis, but differ in their substrate specificity. Some members are highly specific, others have a broad specificity profile. The evolutionary origins and substrate specificities of PONs therefore remain poorly understood. Here, we report a newly identified family of bacterial PONs, and the reconstruction of the ancestor of the three families of mammalian PONs. Both the mammalian ancestor and the characterized bacterial PONX_OCCAL were found to efficiently hydrolyze N-acyl homoserine lactones that mediate quorum sensing in many bacteria, including pathogenic ones. The mammalian PONs may therefore relate to a newly identified- family of bacterial, PON-like "quorumquenching" lactonases. The appearance of PONs in metazoa is likely to relate to innate immunity rather than detoxification. Unlike the bacterial PON, the mammalian ancestor also hydrolyzes, with low efficiency, lactones other than the homoserine lactones, thus preceding the detoxifying functions that diverged later in two of the three mammalian families. The bifunctionality of the mammalian ancestor and the trade-off between the quorum-quenching and detoxifying lactonase activities explain the broad and overlapping specificities of some mammalian PONs versus the singular specificity of others [19]."

\section{Gehring WJ}

"the evolution of vision is retraced from its putative origins in cyanobacteria to humans. Circadian oscillatory clocks, phototropism, and phototaxis require the capability to detect light. Photosensory proteins allow us to reconstruct molecular phylogenetic trees. The evolution of animal eyes leading from an ancestral prototype to the highly complex image forming eyes can be deciphered on the basis of evolutionary developmental genetic experiments and comparative genomics. As all bilaterian animals share the same master control gene, Pax6, and the same retinal and pigment cell determination genes, we conclude that different eyetypes originated monophyletically and subsequently diversified by divergent, parallel, or convergent evolution [20]."

\section{Yi-An Ko et al}

"Epigenetics refers to functionally relevant modifications of the genome that do not involve a change in the nucleotide sequence. Examples of such modifications are DNA methylation and histone modifications. Both this modifications serve to regulate gene expression without altering the underlying DNA sequence. The epigenome encodes critical information to regulate gene expression. The cellular epigenome is established during development and differentiation and maintained during cell division. These instructions are different in each cell type; therefore the epigenome is cell type specific. Nutrient availability and other environmental factors cause changes in the epigenome. Recent studies suggests the critical contribution of the epigenome to the development of complex gene-environmental diseases including chronic kidney diseases.

Epigenetics, meaning 'above (epi-) genetics', is the study of gene expression regulation that cannot be directly attributed to changes in the DNA sequence. Among the 3 billion nucleotides in our genome, less than $2 \%$ are responsible for coding proteins. The rest of the sequences were long thought to be non-functional ('junk'), possibly only there as a buffering zone for mutations. Recent studies, especially those published by the ENCODE consortium, indicate that, by describing and annotating the cellular epigenomes, functional roles could possibly be assigned to as much as $80 \%$ of the genome. It seems that the instruction manual that governs gene expression is hidden somewhere in the 'junk DNA'.

The epigenome involves 'marking' (i.e. chemical modification) of the DNA or associated proteins. Epigenetic marks include cytosine modification (mainly methylation) and histone tail modification. The epigenome is inherited during cell division to maintain cell identity. However, nutrition and other environmental factors change these chemical tags. these chemical tags (i.e. the epigenome) serve as the intersection between the stably inherited genome and the changing environment. Characterization of the epigenome has helped tremendously to define cell-type specific gene expression. A typical transcription unit in a multicellular eukaryote contains both clusters of proximal promoter elements and five types of cisacting regulatory sequences (insulators, promoters, enhancers, silencers, and locus control regions). Insulator areas border and separate transcriptional units. Promoters are usually located at the $5^{\prime}$ end of transcription start site and contain elements called TATA box, which is bound by either a TATA-binding protein or a cluster of cytosine-guanine nucleotide pairs within the linear sequence, known as CpG islands (CGI). For target gene transcription to take place, not only promoters but also long- and short-range regulatory regions are needed. These cis-type gene regulatory regions are highly cell- type specific and are critical for cell-type specific transcription. Simultaneous binding of transcription factors to each other and to the long-and short-range regulatory regions, results in genomic DNA loops that join distant regulatory DNA sequences together [21]."

\section{Joseph E. LeDoux}

"Basic tendencies to detect/respond to significant events are present in the simplest single cell organisms and persist throughout all invertebrates and vertebrates. Within vertebrates, the overall brain plan is highly conserved, though differences in size and complexity also exist. The forebrain differs the most between mammals and other vertebrates. The classic notion that the evolution of mammals led to radical changes such that new forebrain structures (limbic system and neocortex) were added has not held up, nor has the idea that so-called limbic areas are primarily involved in emotion. Modern efforts have focused on the specific emotion systems, like the fear or defense system, rather than on the search for a general purpose emotion systems. Such studies have found that fear circuits are conserved in mammals, including humans. Animal work has been especially successful in determining how the brain detects and responds to danger. Caution 
should be exercised when attempting to discuss other aspects of emotion, namely subjective feelings, in animals since there are no scientific ways of verifying and measuring such states except in humans.

The topic of emotion and evolution typically brings to mind Darwin's classic treatise, Emotions in Man and Animals (Darwin, 1872). In this book Darwin sought to extend his theory of natural selection beyond the evolution of physical structures and into the domain of mind and behavior by exploring how emotions too might have evolved. Particularly important to his kind of argument was the fact that certain emotions are expressed similarly in people around the world, including in isolated areas where there had been little contact with the outside world and thus little opportunity for emotional expressions to have been learned and culturally transmitted. This suggested to him that there must be a strong heritable component to emotions in people. Also important was his observation that certain emotions are expressed similarly across species, especially closely related species, further suggesting that these emotions are phylo-genetically conserved.

With the rise of experimental brain research in the late 19 th century, emotion was one of the key topics that early neuroscientists sought to relate to the brain (LeDoux, 1987). The assumption was that the emotion circuits are conserved across mammalian species, and that it should be possible to understand human emotions by exploring emotional mechanisms in the non-human mammalian brain.

I will first briefly survey the history of ideas about the emotional brain, and especially ideas that have attempted to explain the emotional brain in terms of evolutionary principles. This will lead to a discussion of fear, since this is the emotion that has been studied most thoroughly in terms of brain mechanisms. The chapter will conclude with a reconsideration of what the term emotion refers to, and specifically which aspects of emotion can be studied in animals and which must be studied in humans.

\section{A brief history of the emotional brain: the rise and fall of the limbic system theory}

All organisms, even single cell organisms, must have the capacity to detect and respond to significant stimuli in order to survive. Bacteria, for example, approach nutrients and avoid harmful chemicals. With the evolution of multicellular, metazoan organisms with specialized systems, particularly a nervous system, the ability to detect and respond to significant events increases in sophistication.

Invertebrates, the oldest and largest group of multicellular organisms, exhibit a wide variety of types of nervous systems. However, all vertebrates share a common basic brain plan consisting of three broad zones (hindbrain, midbrain, and forebrain) with conserved basic circuits. In spite of this overall similarity, differences in size and complexity exist. For example, the forebrain differs the most between mammals and reptiles. On the basis of such differences, the classic view of forebrain evolution emerged in the first half of the 20th century. According to this view, with the emergence of mammals, the forebrain plan underwent radical changes in which new structures, especially cortical structures, were added. These were layered over and covered the reptilian forebrain, which mainly consisted of the basal ganglia. First came "primitive" cortical regions in early mammals. In these organisms the basic survival functions related to feeding, defense and procreation were taken care of by fairly undifferentiated (weakly laminated) cortical regions (primitive cortex, including the hippocampus and cingulate cortex) and related subcortical areas (such as the amygdala) that were closely tied to the olfactory system. Later mammals added highly novel, laminated cortical regions (neocortex) that made possible enhanced non-olfactory sensory processing and cognitive functions (including learning and memory, reasoning, and planning capacities, and, in humans, language).

The principle that equated cognition with evolutionarily new cortex (neocortex) and emotion with older cortex and related subcortical forebrain regions culminated in Paul MacLean's limbic system theory of emotion The term limbic was first used by the French anatomist Paul Broca as a structural designation for a rim of cortex in the medial wall of the hemisphere. Broca called this rim the limbic lobe (le grande lobe limbique) (limbic is from the Latin word for rim, limbus). MacLean built on the classic findings of comparative anatomists such as Herrick and Papez, and experimental findings from Walter Cannon, Phillip Bard and Henrich Kluver and Paul Bucy to transform the limbic lobe into an emotion system, the limbic system. The limbic system was defined anatomically as the primitive medial cortical areas and interconnected subcortical nuclei (including the amygdala and septum).

MacLean called the limbic system the paleomammalian brain (since it was said to have emerged with the evolution of early mammals), and contrasted it with the reptilian brain (basal ganglia and brainstem). In more recent mammals the neocortex, also called the neomammalian brain, was said by MacLean to increases in size and complexity at the expense of the limbic system. The decrease of the limbic system reduced the dependence of humans on base emotions, and the increase in the neocortex allowed humans greater control over remaining emotional circuits as well as greater cognitive capacities.

The limbic system concept stimulated much research in the 1950s, 60s, and 70s. However, it has been criticized on a number of grounds, and has been rejected by many scientists. Because the limbic system concept continues to be referred to in some scientific circles and persists in many lay accounts of the brain, it is worth considering why it is not acceptable.

First, the theory presumes that the neocortex and limbic system are unique mammalian specializations. Neither of these ideas is accepted by contemporary comparative neuroanatomists. Birds and reptiles, for example, have been shown to have structures that correspond with both mammalian neocortex and with MacLean's cortical and subcortical limbic areas (hippocampus, amygdala). Second, MacLean argued the architecture of limbic areas is illsuited for cognitive processes. However, the hippocampus, viewed by MacLean as the centerpiece of the limbic system and a central 
structure for emotional functions, is recognized as one of the key areas related to higher cognitive functions, such as declarative or explicit memory and spatial cognition .Third, efforts to define the system have failed. Connectivity with old cortex is a flawed criterion if old cortex is itself an unjustified notion. Connectivity with the hypothalamus once seemed plausible, since that was a way of distinguishing relevant and irrelevant cortical areas (Issacson, 1982). However, as anatomical techniques improved, areas from the neocortex were also found to be connected with the hypothalamus, as were areas of the spinal cord, potentially extending the limbic system across the entire brain. Finally, and perhaps most important, there is no evidence that the limbic system, however defined, functions as an integrated system in the mediation of emotion. While specific areas of the limbic system contribute to some emotional functions, these areas do not do so because they belong to a limbic system that evolved to perform emotional functions. Indeed, relatively few limbic areas have been shown to contribute to emotional functions. As noted above, the hippocampus, the centerpiece of the limbic system theory of emotion, has been strongly implicated in cognitive functions but the evidence for a role in emotion is far less impressive.

The limbic system theory attempted to explain all emotions within a single anatomical concept. Contemporary researchers are more inclined to focus on tasks designed to study the brain systems of specific emotions. this has been a more profitable empirical approach [22]."

\section{Corballis MC}

"The mirror system provided a natural platform for the subsequentevolution of language. In nonhuman primates, the system provides for the understanding of biological action, and possibly for imitation, both prerequisites for language. I argue that language evolved from manual gestures, initially as a system of pantomime, but with gestures gradually "conventionalizing" to assume more symbolic form. The evolution of the episodic memory and mental time travel, probably beginning with the genus Homo during the Pleistocene, created pressure for the system to "grammaticalize," involving increased vocabulary necessary to refer to episodes separated in time and place from the present, constructions such as tense to refer to time itself, and the generativity to construct future (and fictional) episodes. In parallel with grammaticalization, the language medium gradually incorporated facial and then vocal elements, culminating in autonomous speech (albeit accompanied still by manual gesture) in our own species, Homo sapiens [23]."

\section{Charles C. Horn}

"Nausea -vomiting are important as biological systems for drug side effects, disease co-morbidities, and defenses against food poisoning. Vomiting can serve the function of emptying a noxious chemical from the gut, and nausea appears to play a role in a conditioned response to avoid ingestion of offending substances. The sensory- pathways for nausea and vomiting, such as gut and vestibular inputs, are generally defined but the problem of determining the brain's final common pathway and central pattern generator for nausea and vomiting is largely unsolved.
A neurophysiological analysis of brain pathways provides an opportunity to more closely determine the neurobiology of nausea and vomiting and its prodromal signs (cold sweating, salivation).

Nausea - vomiting are commonly studied at pharmacological, behavioral, and psychological levels of analysis. These approaches are represented by a large literature of human clinical research highlighting the efficacy of various anti-emetic agents. Extensive work has also been conducted to demonstrate that treatments for disease do not have negative effects, such as nausea and vomiting, that might limit their clinical application. The current scarcity of research on the neurobiological basis of nausea and vomiting is striking considering its clinical importance. For example, at the 2006 annual meeting of the Society for Neuroscience there were $>14,000$ presentation abstracts but only 19 contained the words vomiting, emesis, or nausea.

This review presents nausea and vomiting in the evolutionary context of food intake (i.e., what is the adaptive nature of these systems?), discusses the relevance of this topic to today's world, and addresses the current understanding of the brain circuitry that generates nausea and vomiting.

\section{Nausea and vomiting: Defenses against food poisoning}

Animals possess an arsenal of special abilities for survival and many of these are used for the foraging and consumption of food. Food intake is a risky behavior leading to the exposure of internal organs to possible food-related ailments, including viral and bacterial infection, allergies, and food intolerance. An important survival problem is to determine which foods are safe and animals possess a hierarchy of sensory systems that help in food identification. Many spoiled foods can be identified using olfactory cues and taste is an effective intake deterrent when food is sour or bitter.

Smell and taste, the gatekeepers of the alimentary tract, are not always effective in detecting the quality of food, and nausea and vomiting, as additional mechanisms for dealing with an unhealthy meal, play a large role in subsequent levels of defense. Emesis, along with diarrhea, helps rid the gastrointestinal tract of dangerous ingested toxins. The vomiting response is present in many species, appearing in most vertebrates (including representative members of fish, amphibia, reptiles, birds, and mammals and at least one invertebrate, the gastropod pleurobanchaea .However, the broad assessment of the emetic response across species is hampered by the problem of distinguishing emesis from processes of regurgitation and rumination; emesis is functionally different and likely represents a more forceful ejection of gastric contents.

Several commonly used laboratory animals appear to lack a vomiting response (e.g., rat, mouse, guinea pig, and rabbit). It is worth noting however that only a few strains of these species have been tested for emesis, using a limited set of stimuli, and it is unknown whether all members of these species lack the response. The possibility exists that rodents possess a degenerate "emetic" response rather than an absent one. There is an isolated report of "retching" in mice (Furukawa \& Yamada, 1980) and rats have a gag reflex, which has similar features to a single retch, triggered 
by mechanical stimulation of the pharynx. There are structural differences in the rat and mouse esophagus and diaphragm that would make it difficult to generate the emetic response. Perhaps the vomiting response became an unneeded level of protection in rodents because they possess other efficient ways to deal with potential toxicosis, including a finely tuned ability to develop conditioned flavor aversions (CFA).

Nausea is an aversive experience that often accompanies emesis, and is a distinct perception, different from pain or stress. Although a rare condition, vomiting can occur without nausea .Nausea is not simply the result of a low level of stimulation to the emetic system, which if only increased in intensity would result in vomiting. Counter-intuitively, nausea is more difficult to treat than emesis using anti-vomiting medications. The severity of druginduced emesis (e.g., from cancer chemotherapy) can be controlled with anti-emetic medications, such as 5-HT3 and NK1 receptor antagonists; but nausea is still a persistent problem These facts suggest that nausea and vomiting are at least partially separate physiological processes. Arguably, nausea is the driving force behind the development of CFA - thus providing the potent unconditioned stimulus to support a learned response to avoid consumption of foods which make us sick Unfortunately, nausea is difficult to study in laboratory animals but animal behavior (salivation, conditioned aversion), under conditions that make humans nauseated, suggests the presence of a unique aversive state.

Pregnancy-induced nausea and vomiting has an adaptive advantage. Importantly, the first trimester is a period of rapid fetal growth, and includes critically the development of the CNS, which is highly susceptible to toxicosis. Pregnant women also appear to be picky eaters during this period and tend to avoid meat and fish products, which are more likely to contain pathogens that might harm the fetus. In humans, the presence of pregnancyinduced nausea and vomiting in the first trimester is correlated with a healthy pregnancy. It is only in rare cases that pregnancyinduced nausea and vomiting extends beyond this time interval, compromising the health of mother and fetus; a condition called hyperemesis gravidarum [24]."

\section{Cloninger CR}

The functional structure of self-aware consciousness in human beings is described based on the evolution of human brain functions. Prior work on heritable temperament and character traits is extended to account for the quantum-like and holographic properties (i.e. parts elicit wholes) of self-aware consciousness. Cladistic analysis is used to identify the succession of ancestors leading to human beings. The functional capacities that emerge along this lineage of ancestors are described. The ecological- context in which each cladogenesis occurred is described to illustrate the shifting balance of evolution as a complex adaptive system. Comparative neuroanatomy is reviewed to identify the brain structures and networks that emerged coincident with the emergent brain functions. Individual differences in human temperament traits were well developed in the common ancestor shared by reptiles and humans. Neocortical development in mammals proceeded in five major transitions: from the early reptiles to early mammals, early primates, simians, early Homo, and modern Homo sapiens. These transitions provide the foundation for human self-awareness related to sexuality, materiality, emotionality, intellectuality, and spirituality, respectively. The functional structure of human selfaware consciousness is concerned with the regulation of five planes of being: sexuality, materiality, emotionality, intellectuality, and spirituality. Each plane elaborates neocortical functions organized around one of the five special senses. The interactions among these five planes gives rise to a $5 \times 5$ matrix of subplanes, which are functions that coarsely describe the focus of neocortical regulation. Each of these 25 neocortical functions regulates each of five basic motives or drives that can be measured as temperaments or basic emotions related to fear, anger, disgust, surprise, and happiness/ sadness. The resulting $5 \times 5 \times 5$ matrix of human characteristics provides a general and testable model of the functional structure of human consciousness that includes personality, physicality, emotionality, cognition, spirituality in a unified developmental framework [25].

\section{Javier DeFelipe}

"The tremendous expansion and the differentiation of the neocortex constitute 2 major events in the evolution of the mammalian brain. The increase in size and complexity of our brains opened the way to a spectacular development of cognitive and mental skills. This expansion during evolution facilitated the addition of microcircuits with a similar basic structure, which increased the complexity of the human brain and contributed to its uniqueness. Fundamental differences even exist between distinct mammalian species. Here, we shall discuss the issue of our humanity from a neurobiological and historical perspective.

Homo sum, humani nihil a me alienum puto (I am a human being, and therefore, nothing human is strange to me) Publius Terentius Afer (195/185-159 BC).

The nervous -system has evolved over millions of years, generating a wide variety of species-specific brains and behavioral capacities. For example, the production and appreciation of art seems to be a uniquely human attribute, a recently acquired cognitive capacity in the genus Homo. Almost everything that the human being creates has a touch of art, although we do not need beauty or an esthetic perception to survive but rather, it just simply produces intellectual pleasure. The same occurs with other mental activities, like reading a book / listening to music. It seems obvious that only anatomically modern humans (Homo sapiens) can be behaviorally modern, capable of creating symbolic objects.

Maybe this is when we discovered the world of ideas and created the concept of the soul or spirit. From that moment, the relentless pursuit to define where such a trait is forged began, resulting in the so called "mind-body problem." Of the numerous images available, we have chosen two here to illustrate in distinct ways the relationship between the mental and the physical worlds, both suggesting a separation between the two entities. Perhaps modern neuroscience has contributed most in this field by addressing the issue of mental processes from a biological standpoint. Nevertheless, it is striking how little influence this neuroscientific knowledge has had on society due to the failure in conciliating the 
relationship between the brain and our humanity. It is commonly thought that the increase in complexity as our brain has evolved is a product of the addition of microcircuits with a similar basic structure that incorporate only minor variations. Indeed, speciesspecific behaviors may arise from very small changes in neuronal circuits. However, we will see that the human cerebral cortex has some distinctive circuits that are most likely related to our humanity. In addition, there are some erroneous popular beliefs regarding the relationship between brain size, evolution, and intellectual capabilities, and regarding the patterns of convolutions and the external morphology of the brain. Here, I shall deal with these topics with the aid of some historical notes [26]".

\section{Jon H. Kaas}

"The large size and complex organization of the human brain makes it unique among primate brains. In particular, the neo-cortex constitutes about $80 \%$ of the brain, and this cortex is subdivided into a large number of functionally specialized regions, the cortical areas. Such a brain mediates accomplishments and abilities unmatched by any other species. How did such a brain evolve? Answers come from comparative studies of the brains of present-day mammals and other vertebrates in conjunction with information about brain sizes and shapes from the fossil record, research of brain development, and principles derived from studies of scaling and optimal design. Early mammals were small, with a small brains, an emphasis on olfaction, and little neocortex. Neocortex was transformed from the single layer of output pyramidal neurons of the dorsal cortex of earlier ancestors to the six layers of all present-day mammals. This small cap of neocortex was divided into 20-25 cortical areas, including primary and some secondary sensory areas that characterize neocortex in nearly all mammals today. Early placental mammals had a corpus callosum connecting the neocortex of the two hemispheres, a primary motor area, M1, and perhaps one or more premotor areas. One line of evolution, Euarchontoglires, led to present-day primates, tree shrews, flying lemurs, rodents and rabbits. Early primates evolved from small-brained, nocturnal, insect-eating mammals with an expanded region of temporal visual cortex. These early nocturnal- primates were adapted to the fine branch niche of the tropical rainforest by having an even more expanded visual system that mediated visually guided reaching and grasping of insects, small vertebrates, and fruits. Neocortex was greatly expanded, and included an array of cortical areas that characterize neocortex of all living primates. Specializations of the visual system included new visual areas that contributed to a dorsal stream of visuo-motor processing in a greatly enlarged region of posterior parietal cortex and an expanded motor system and the addition of a ventral premotor area. Higher visual areas in a large temporal lobe facilitated object recognition, and frontal cortex, included granular prefrontal cortex. Auditory- cortex included the primary and secondary auditory areas that characterize prosimian and anthropoid primates today. As anthropoids emerged as diurnal primates, the visual system specialized for detailed foveal vision. Other adaptations included expansion of prefrontal cortex and insular cortex. The human and chimpanzee-bonobo lineages diverged some 6, 8 million years ago with brains that were about one-third the size of modern humans. Over the last 2 million years, the brains of our more recent ancestors increased greatly in size, especially in the prefrontal, posterior parietal, lateral temporal, and insular regions. Specialization of the two cerebral hemispheres for related, but different functions became pronounced, and language and other impressive cognitive abilities emerged [27]."

\section{Harvey J. Karten}

"The organization of the non-mammalian forebrain had long puzzled neurobiologists. Unlike typical mammalian brains, the telencephalon is not organized in a laminated 'cortical' manner, with distinct cortical areas dedicated to individual sensory modalities or motor functions. The 2 major regions of the telencephalon, the basal ventricular ridge and the dorsal ventricular ridge, were loosely referred to as being akin to the mammalian basal ganglia. The telencephalon of non-mammalian vertebrates appears to consist of multiple 'subcortical' groups of cells. Analysis of the nuclear organization of the avian brain, its connections, molecular properties and physiology, and organization of its pattern of circuitry and function relative to that of mammals, collectively referred to as 'evolutionary connectomics', revealed that only a restricted portion of the BVR is homologous to the basal ganglia of mammals. The remaining dorsal regions of the DVR, wulst and arcopallium of the avian brain contain telencephalic inputs and outputs remarkably similar to those of the individual layers of the mammalian 'neocortex', hippocampus and amygdala, with instances of internuclear connections strikingly similar to those found between cortical layers and within radial 'columns' in the mammalian sensory and motor cortices. The molecular properties of these 'nuclei' in birds and reptiles are similar to those of the corresponding layers of the mammalian neocortex. The fundamental path-ways and cell groups of the auditory, visual and somatosensory systems of the thalamus and telencephalon are homologous at the cellular, circuit, network and gene levels, and are of great antiquity. A proposed altered migration of these homologous neurons and circuits during development is offered as a mechanism that may account for the altered configuration of mammalian telencephalae" [28].

\section{Robert K. Naumann et al}

"'Deep inside the skull of every one of us there is something like a brain of a crocodile. Surrounding the R-complex is the limbic system or mammalian brain, which evolved tens millions of years ago in ancestors who were mammal but not yet primates. It is a major source of our moods and emotions, of our concern and care for the young. Living in uneasy truce with the more primitive brains beneath, is the cerebral cortex; civilization is a product of the cerebral cortex."

“Carl Sagan's amusing words of wisdom notwithstanding is the H-bomb not also a product of the cerebral cortex? - is the reptilian brain really just a mammalian brain missing most of the parts? Some 320 million years ago, the evolution of a protective membrane surrounding the embryo, the amnion, enabled vertebrates to develop outside of water and thus to invade new terrestrial niches. These amniotes were the ancestors of today's mammals and sauropsids (reptiles, birds). Present-day reptiles 
are a diverse group of more than 10,000 species that comprise sphenodons ('Tuatara'), lizards, snakes, turtles and crocodilians. Although turtles were once thought to be the most 'primitive' among reptiles, current genomic data point toward two major groupings: the Squamata (lizards and snakes); a group comprising both the turtles and the Archosauria (dinosaurs, modern birds and crocodilians). Dinosaurs inhabited the Earth from the Triassic (230 million years ago), at a time when the entire landmass formed a single Pangaea. They flourished from the beginning of the Jurassic to the mass extinction at the end of the Cretaceous ( 65 million years ago), and birds are their only survivors.

\section{Vertebrate phylogeny, gross brain morphology, and homologous regions in the forebrain}

(A) Phylogenetic tree of vertebrates and timeline of major events in amniote evolution. Shaded area in the phylogenetic tree indicates 'reptiles' as defined in the text. Red lines (lower panel) indicate the origin of amniotes (320 million years ago), the origin of dinosaurs (230 million years ago) and their extinction (65 million years ago). Schematic drawings showing brains (lateral view, anterior left) of vertebrate representatives: from top to bottom, a fish (knife-fish), an amphibian (tiger salamander), a reptile (monitor lizard), a bird (pigeon) and a mammal (hedgehog tenrec). Major subdivisions (examples in color) are present in all species but appear in different proportions. (B) Transverse section of the right hemisphere of vertebrate representatives: from top to bottom, a fish (zebrafish), an amphibian (frog), a reptile (lizard), a bird (pigeon) and a mammal (rat). Colors represent conserved pallial subdivisions.

What we generally call reptiles is thus a group defined in part by exclusion: it gathers amniote species that are neither mammals nor birds (Figure 1), making the reptiles technically a paraphyletic grouping. Despite this technical point, the so-defined reptiles share many evolutionary, anatomical, developmental, physiological (for example, ectothermia), and functional features. It is thus reasonable to talk about a 'reptilian brain' — the subject of this Primer.

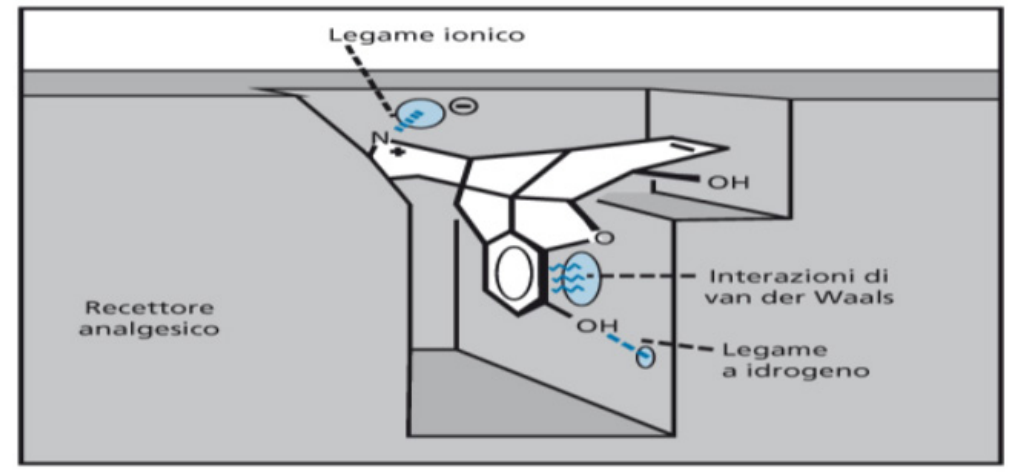

FIGURA 24.7 Interazioni di legame della morfina con il sito di legame del recettore oppioide.

Graham L. Patrick

Limica Farmaceutica

EdisES

Figure 1: Oppiaceus Human receptor: SAR structure activity relationship.

\section{Structure and evolution of the reptilian brain}

The diversity of reptiles and their evolutionary relationship to mammals make reptilian brains great models to explore questions related to the structural and functional evolution of vertebrate neural circuits. To this end, comparative research studies seek to identify homologies - structural or molecular similarities that are due to common ancestry - at a variety of levels, for example, brain regions, circuits or cell types. Homologies can be inferred from extant species by using a comparative approach within a phylogenetic framework. Vertebrate brains have been classically compared in terms of morphology, connectivity, and neurochemistry; however, adult neuro-anatomy may not be sufficient to determine homologies without ambiguity.

Vertebrates share among themselves the highest morphological resemblance not as adults, but at their so-called 'phylotypic stage' of embryonic development, a resemblance mirrored by similarity across transcriptomes. Identification of conserved brain subdivisions, established by conserved signaling centers and uniquely defined by the combinatorial expression of transcription factors during development, demonstrates that all of the general brain regions found in mammals, including the cerebral cortex, have homologies in reptiles. The cerebral cortex is part of the pallium, a developmental subdivision of the telencephalon delineated by the expression of transcription factors such as Pax6, Emx1 and Tbr1, which is conserved in all vertebrates (Figure 2). Gene expression data show that the same fundamental subdivisions of the pallium - lateral, ventral, medial and dorsal, the latter giving rise to the neocortex in mammals - occur in developing vertebrates, despite the divergent morphologies of pallial structures in adults. The cerebral cortex is thus not a mammalian invention, but rather an ancient pallial structure that predates the split between sauropsids and theropsids, the mammals' precursors. 


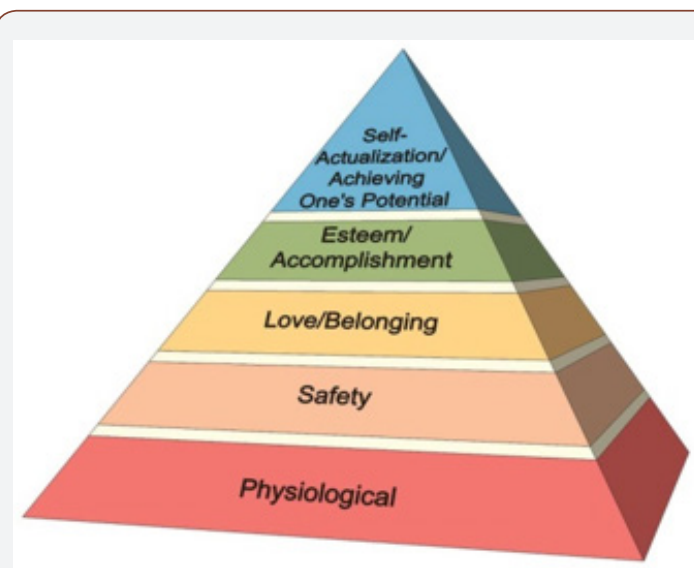

ES:

Figure 2: Maslow's Hierarchy of Needs

\section{Developmental and adult bauplan of the vertebrate brain}

Simplified developmental scheme of vertebrate brain regions. Combinatorial expression of transcription factors such as Tbr1, Dlx5, and Gbx2 defines brain regions during development and sets the stage for further differentiation in adults. Note that Tbr1 and Dlx5 delineate further regions not shown here.

Lateral and medial pallium express different sets of molecular markers during development. Which regions of the adult brain correspond to lateral and medial pallium is an active topic of research and thus we combine both pallial subdivisions into a single region.

Comparative studies of brain structure and development have revealed a general Bauplan, an outline of the fundamental large-scale architecture of the vertebrate brain, reflecting its basic functional organization. The telencephalon integrates and stores multimodal information and is also the higher center of action selection and motor control (basal ganglia). The hypothalamus is a conserved area controlling homeostasis and behaviors essential for survival, such as feeding and reproduction. In all vertebrates, behavioral states are controlled by common brainstem neuromodulatory circuits, such as the serotoninergic system. Finally, vertebrates harbor a diverse set of sense organs, and their brains share pathways for processing incoming sensory inputs. For example, in all vertebrates, visual information from the retina is relayed and processed to the pallium through the tectum and the thalamus, while olfactory input from the nose first reaches the olfactory bulb and then the pallium.

Although pallial structures exist in amphibians and fish, reptiles and mammals are the only vertebrates to have a cerebral cortex with a clear, though simple, three-layered structure, similar to that of mammalian allocortex. The reptilian ventral pallium also gives rise to the dorsal ventricular ridge, a structure that dominates the bird pallium and contributes to the complex cognitive abilities of birds, but whose mammalian equivalent is still the subject of debate among comparative anatomists. The reptilian cortex contains far fewer subdivisions than that of rodents, carnivores, or primates: it is subdivided into a medial cortex, often called hippocampus by anatomists; a lateral cortex, equivalent to the mammalian piriform cortex; and a dorsal cortex in between, which receives multimodal inputs (for example, visual inputs in turtles). There is an evidence for motor and somatosensory areas in the reptilian cortex, but pallial motor control may have evolved early in vertebrate evolution. Owing to this simplicity (Figure 3), the reptilian brain facilitates the study of primordial cortical function as a whole, and points to the origins of cortex as fulfilling general associative functions.

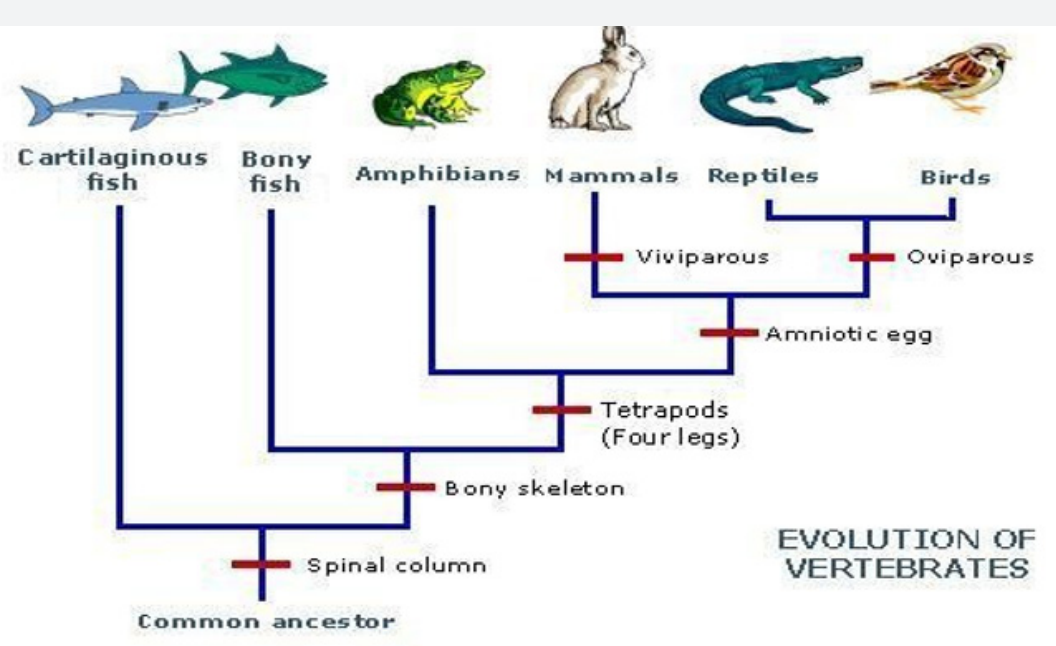

Figure 3: Evolution of vertebrates.

\section{Visual circuits in monkey and turtle}

Comparison of known visual cortical circuits between macaque and freshwater turtle, emphasizing the great simplicity of reptilian cortex. Arrow indicates direction of information flow from sensory periphery. aDVR, anterior dorsal ventricular ridge (a: auditory, s: somatosensory, v: visual); dLGN, dorsal lateral geniculate nucleus; D1 and D2, dorsal cortex areas D1 and D2; DM, dorsomedial cortex; M (HC), medial cortex ('hippocampus'); N.Rot., nucleus rotundus ('pulvinar'); OT, optic tectum ('superior colliculus'); RGC, retinal ganglion cells. 
Besides sharing pallial modules, mammals and reptiles also share a complement of cortical cell types, suggesting that some structural elements of cortical circuits arose early in amniote evolution. Like the mammalian cortex, the reptilian cortex contains excitatory, glutamatergic neurons and inhibitory (GABAergic) interneurons. In both mammals and reptiles, these neurons have a common developmental origin: excitatory neurons are generated by multipotent cortical progenitors, whereas inhibitory neurons are born in subpallium before migrating to the cortex.

Classical studies suggest that the reptilian main cortical cell layer (layer 2, L2) corresponds to the deep, output layers of mammalian neocortex, whereas its layer 1 (L1) is equivalent to mammalian layer I. According to this view, mammalian cortical evolution would have included the incorporation of new, intermediate cell layers acting as input stations and internal circuitry. Morphologically, L2 pyramidal neurons of the reptilian dorsal cortex are most similar to mammalian hippocampal excitatory neurons. Indeed, reptilian pyramidal neurons have, depending on the area, little to no basal dendritic field, and several densely spine-studded apical dendrites, quite different from the single, long, apical dendrite of neocortical pyramidal neurons. Consistent with this correspondence between layers, reptilian sub-pallial cells transplanted into mammalian embryos generate GABAergic neurons that can populate only the deeper cortical layers.

Challenging this view recent molecular studies have found that turtle and lizard cortical neuro-blasts generate neurons that express upper layer molecular markers, in a developmental sequence similar to that observed in mammals. Although the molecular characterization of neuronal types in the reptilian cortex is still in its infancy, it is possible that the reptilian cortex represents an ancestral blueprint for the more elaborate mammalian cortical circuits. Reptilian cortical neurons, or subsets of them, might share molecular (and functional) features with both upper and lower layer mammalian cells.

\section{Cortical Function}

Cortical circuitry is where most of the comparative work on the reptilian brain has been done. In the three-layered reptilian cortex, afferent inputs travel medially through superficial L1, where they fan out in a non-topographic manner. Their targets are GABAergic interneurons (with cell bodies in L1, L3) and glutamatergic pyramidal neurons, with cell bodies packed in L2, and dendrites in L1 , L3. Interneurons project locally within cortex, providing feed forward and feedback inhibition to specific regions of the pyramidal cells' dendrites. Several interneuron subtypes can be defined based on the expression of a subset of common genetic markers used in mammals. in turtle cortex, some interneuron types express calbindin, others express neuropeptide-Y, while parvalbumin (PV)positive interneurons appear to be absent. Pyramidal cells make reciprocal connections with each other and with interneurons locally, with subcortical afferent structures, and with other cortical areas.

The lateral cortex receives olfactory input from the olfactory bulb, and projects to the medial cortex (hippocampus). The dorsal cortex receives input from the thalamus; in many species this input is visual, originating in the thalamic lateral geniculate nucleus and also eventually reaches the hippocampus. Pyramidal cells of the hippocampus project back to dorsal and lateral cortices, forming an internal cortical loop. Different mammalian species can exhibit vast elaborations in the number and connectivity of cortical subregions. By examining homologous structures, shared circuit motifs can be recognized.

\section{Shared circuit motifs between reptilian and mammalian cortex}

Whereas processing steps are fewer in reptiles, they reach the same target as in mammalian cortex. Cortical architecture is more similar across regions in reptiles, suggesting similar and possibly general computations are performed on different sensory inputs.

In turtles, visual stimulation triggers propagating waves of neural activity that travel across the cortex. These waves are slower and simpler than those observed in mammalian neocortex. They are accompanied by relatively slow oscillations, which are most prominent in the $20 \mathrm{~Hz}$ frequency band. Whereas the so-called gamma oscillations in mammalian cortex are typically around and above $40 \mathrm{~Hz}$, recent results in mice indicate that the $20 \mathrm{~Hz}$ band dominates when PV-interneuron development is artificially arrested, consistent with the above observation that turtle cortex lacks PV interneurons. The computational role, if any, of such dynamics is unknown at present. Progress will require new experimental approaches that allow the simultaneous sampling of large neuronal populations. Specific and data-driven theories of computation in reptilian cortex thus await further study. To the extent that modern reptilian cortex resembles that in the common ancestor of reptiles and mammals, understanding reptilian cortex function may reveal some of the fundamental associative computations that early cortical circuits evolved to carry out [29]."

\section{Wynn T et al writed}

"How did the human mind evolve? How and when did we come to think in the ways we do? The last thirty years have seen an explosion in research related to the brain and cognition. This research has encompassed a range of biological and social sciences, from epigenetics /cognitive neuroscience to social - developmental psychology. Evolutionary scholars, including paleoanthropologists, have deployed the standard array of evolutionary methods. Ethological and experimental evidence has added significantly to our understanding of nonhuman brains and cognition, especially those of nonhuman primates. Studies of fossil brains through endocasts and sophisticated imaging techniques have revealed evolutionary changes in gross neural anatomy. Psychologists have also gotten into the game through application of reverse engineering to experimentally based descriptions of cognitive functions. For the hominin evolution, there is another rich source of evidence of cognition, the archeological record. Using the methods of Paleolithic archeology and the theories and models of cognitive science, evolutionary cognitive archeology documents developments in the hominin mind that would otherwise be inaccessible [30]."

\section{According Averof $M$ et al}

"2 hypotheses have been proposed for the origin of insect wings. One holds that wings evolved by modification of limb 
branches that were already present in multibranched ancestral appendages and probably functioned as gills. The second proposes that wings arose as novel outgrowths of the body wall, not directly related to any pre-existing limbs. If wings derive from dorsal structures of multibranched appendages, we expect that some of their distinctive features will have been built on genetic functions that were already present in the structural progenitors of insect wings, and in homologous structures of other arthropod limbs. We have isolated crustacean homologues of two genes that have wing-specific functions in insects, pdm (nubbin) and apterous. Their expression patterns support the hypothesis that insect wings evolved from gill-like appendages that were already present in the aquatic- ancestors of both crustaceans and insects" [31].

\section{B.J. PEARSON et al showed that}

"All multi-cellular organisms have requirements for tumor suppression to regulate cellular proliferation during either embryonic development or adult life. Different organisms have vastly different requirements. Adult tumor suppression is probably not crucial to organisms possessing both short life spans and largely postmitotic soma. In contrast, animals with lifelong tissue turnover or those capable of regenerating body parts lost to injury must possess evolutionarily selected mechanisms to control rates of cell proliferation such that tissue homeostasis can be maintained or restored after injury. We hypothesize that these differences may help to explain why the lists of tumor suppressor genes in humans and Drosophila are largely nonoverlapping. Here, we address this disparity by examining the tumor suppressor gene content of two outgroups to the vertebrates and flies/nematodes: the freshwater planarian and the single-celled choanoflagellate. Both organisms have recently had their genomes sequenced, giving us a first glimpse of which known tumor suppressor genes have been maintained during evolution. In addition, we attempt to resolve which genes may have had ancestral tumor suppressor function and which may have acquired this function de novo [32]."

\section{Hojung Nam et al}

"Evolution results from molecular-level changes in an organism, thereby producing novel phenotypes and, eventually novel species. Changes in a single gene can lead to significant changes in biomolecular networks through the gain and loss of many molecular interactions. Thus, significant insights into microbial evolution have been gained through the analysis and comparison of reconstructed metabolic networks. However, challenges remain from reconstruction incompleteness and the inability to experiment with evolution on the timescale necessary for new species to arise. Despite these challenges, experimental laboratory evolution of microbes has provided some insights into the cellular objectives underlying evolution, under the constraints of nutrient availability and the use of mechanisms that protect from extreme conditions.

While evolution involves the change of genetic sequence over time, it more importantly changes molecular interactions. Therefore, evolution often leads to modifications in molecularnetwork topology and ultimately in the systems-level functions of an organism (phenotype).
Metabolism, a complex network of chemical reactions, is an ideal system to study network-level changes in evolution. it is the best characterized bio-molecular network. The underlying genes and proteins are well-characterized, and their interactions and biochemistry have been studied comprehensively. Because metabolism is critical to all cellular functions, many core reactions have ancient origins and are conserved across all kingdoms of life. While small differences exist in metabolic pathway topology within different strains of one species (in Pseudomonas syringae ), examination of conserved reactions across species nonetheless facilitates clear comparisons for evolutionary insight.

Selective pressures resulting from cellular objectives help guide the expansion and pruning of metabolic pathways through evolution. The cellular objectives underlying the selective pressure can involve maximizing the nutritional potential of the environment and surviving environmental stress. Here we review recent insights into metabolic pathway evolution, which have been gained from both computational and experimental approaches"[33] (Figures 4-8).

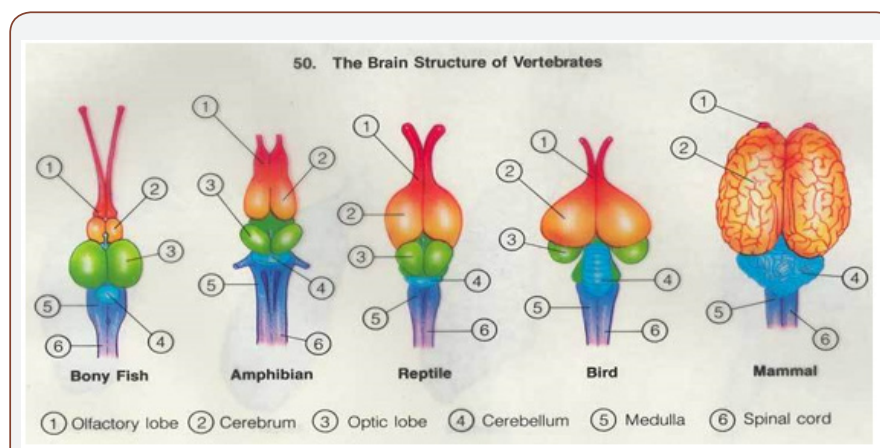

Figure 4: Brain structure in vertebrate.

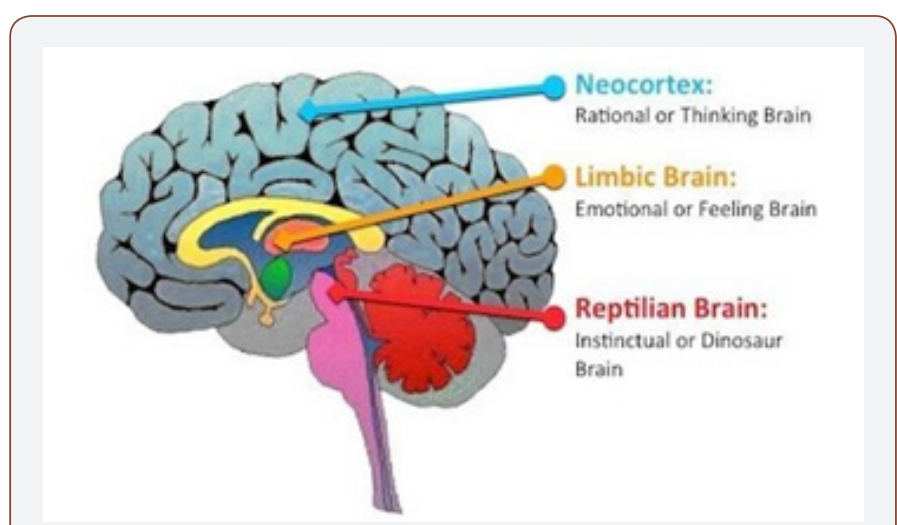

Figure 5: Reptilian, limbic brain and neocortex.

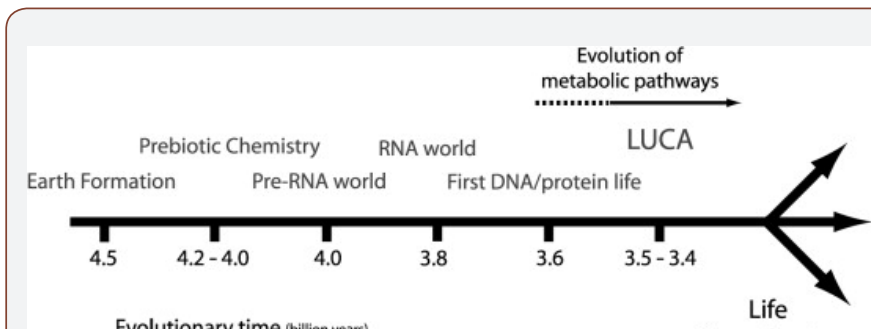

Evolutionary time (billion years) diversification

Figure 6: Metabolic pathways evolution. 


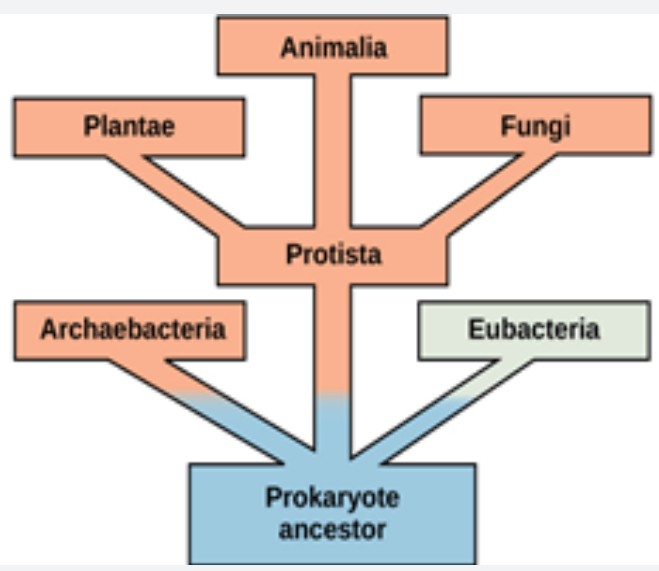

Figure 7: Evolution of life.

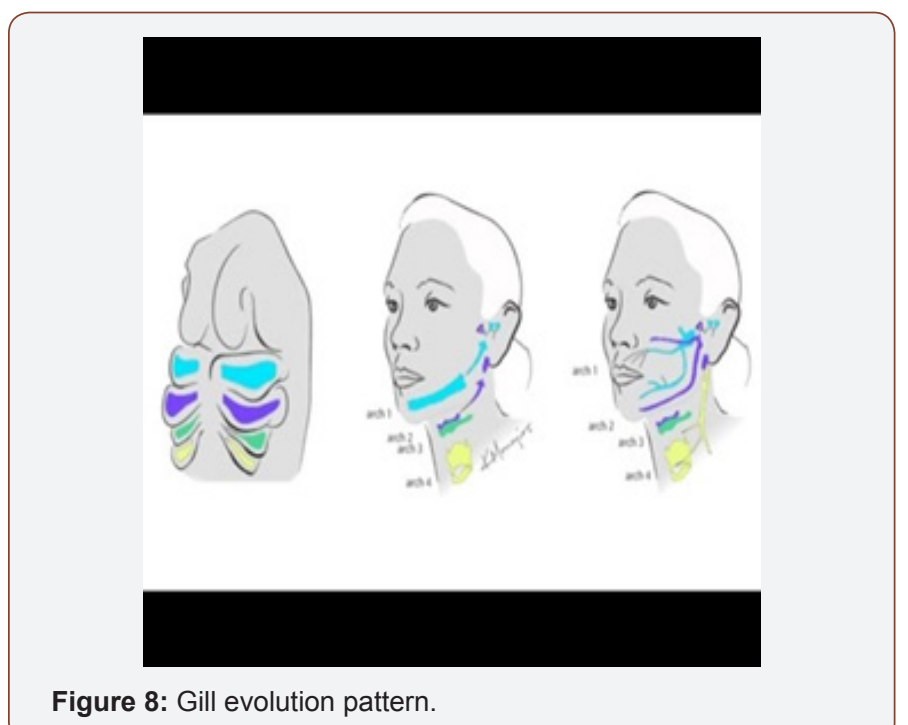

\section{Discussion}

Related the results of reporter scientific literature analized in a unic way We propose to the scientific world to introduce a new discipline named ENDOGENOUS ARCHEOLOGY.

To collect and rationalize all the knowledge related to this topics to create a unic database and source of information for future investigations.

Residual organs, genes, metabolic pathways, immune response, ancestral systems can be observed in actual human being as an archeological ruins to testify an evolutive process.

Emotional control, fear, aggressivity, sexual and surviving instinct, some immune process, onco suppressor and and many other Functions are strictly related with a common evolution and with real objectivable evidence.

\section{Conclusion}

Related to the result $\mathrm{f}$ this review- research work is possible to submit to the researcher community a new systematic discipline to collect, rationalize and join many previous and actual distinct theory in a new discipline.

A new discipline that can make possible to open at new research modalities.
Archeological evidence inside us to be managed as archerological finding.

Evidence like emotional management by humans are strictly related to the neuro- receptorial profile.

Can be used as traceant to investigate ancestral circuits.

A starting and in progress researchgate project (opened in November 2018) can be the right instrument to rationalize all scientific evidence produced since this time for future research.

A deep understand in our past can be really useful in scientific new application in many different field like.

Behavioral science, psychological but also in biology and medicine, pharmacology, immunology and other relevant field and also in order to introduce new diagnostic or therapeutics strategies.

The use of a Filogenetic and Archeo-biological endogenous approach make possible to achieve better scientific results in many discipline.

\section{Acknowledgement}

None.

\section{Conflict of Interest}

This work is produced without any diagnostic or therapeutic intent, only to suggest new research hypotesys and methods.

\section{References}

1. Mauro Luisetto, Khan FA, Cabianca L, Mokbul MI, Rafa AY, et al. (2016) Amygdala pharmacology and crime behavior, dysfunctions to be considered as a disease. Int Arch Bio Med Clin Res 2(2): 1-4.

2. Luisetto M, Mashori GR, Nili-Ahmadabadi B, Khan FA, Khan KR (2018) Mindset kinetics and some depression status: A new quantitative model under biochemical - toxicology approach?. Insights on the Depression and Anxiety 2: 029-039.

3. Luisetto M (2017) Brain and transmission signal modulation Short Communication. Theranostics of Brain Disorders 1(1): 001

4. Luisetto Mauro (2017) Jurisdictional Consequences in some brain condition.

5. Luisetto M, Behzad Nili-Ahmadabadi, Ghulam Rasool Mashori, Ahmed Yesvi Rafa, et al. (2018) Brain and Immune System: KURU, a Strange Kind of Disease. An Endogenous Toxicological Process Like? EC Neurology 10(7): 613-625.

6. Carey HV, Andrews MT, Martin SL (2003) Mammalian hibernation: cellular and molecular responses to depressed metabolism and low temperature. Physiol Rev 83(4): 1153-1181.

7. Davidson I (2010) The archeology of cognitive evolution. Wiley Interdiscip Rev Cogn Sci 1(2): 214-229.

8. Neubauer S, Hublin JJ, Gunz P (2018) The evolution of modern human brain shape. Sci Adv 4(1): eaao5961.

9. Bonett RM (2016) Analyzing endocrine system conservation and evolution. Gen Comp Endocrinol 234: 3-9.

10. Nunn CL, Samson DR, Krystal AD (2016) Shining evolutionary light on human sleep and sleep disorders. Evol Med Public Health 16(1): 227243.

11. Müller M (1992) Energy metabolism of ancestral eukaryotes: a hypothesis based on the biochemistry of amitochondriate parasitic protists. Biosystems 28(1-3): 33-40.

12. Fondi M, Brilli M, Emiliani G, Paffetti D, Fani R (2007) The primordial metabolism: an ancestral interconnection between leucine, arginine, and lysine biosynthesis. BMC Evol Biol 7Suppl 2: S3. 
13. Farhud DD, Zarif Yeganeh M (2013) A brief history of human blood groups. Iran J Public Health 42(1): 1-6.

14. de Boer B (2017) Evolution of speech and evolution of language. Psychon Bull Rev 24(1): 158-162.

15. Heyes C (2012) New thinking: the evolution of human cognition. Philos Trans R Soc Lond B Biol Sci 367(1599): 2091-2096.

16. Thrall PH, Bever JD, Burdon JJ (2010) Evolutionary change in agriculture: the past, present and future. Evol Appl 3(5-6): 405-408.

17. Luca F1, Perry GH, Di Rienzo A (2010) Evolutionary adaptations to dietary changes. Annu Rev Nutr 30: 291-314.

18. Tattersall I (2010) Human evolution and cognition. Theory Biosci $129(2-$ 3): 193-201.

19. Bar-Rogovsky H, Hugenmatter A, Tawfik DS (2013) The evolutionary origins of detoxifying enzymes: the mammalian serum paraoxonases (PONs) relate to bacterial homoserine lactonases. J Biol Chem 288(33):23914-23927.

20. Gehring WJ (2014) The evolution of vision. Wiley Interdiscip Rev Dev Biol 3(1): 1-40

21. Ko YA, Susztak K (2013) Epigenomics: the science of no-longer-junk DNA. Why study it in chronic kidney disease? Semin Nephrol 33(4): 354-362.

22. LeDoux JE (2012) Evolution of human emotion: a view through fear. Prog Brain Res 195: 431-442.

23. Corballis MC (2010) Mirror neurons and the evolution of language. Brain Lang 112(1): 25-35.
24. Horn CC (2008) Why is the neurobiology of nausea and vomiting so important? Appetite 50(2-3): 430-434.

25. Cloninger CR (2009) Evolution of human brain functions: the functional structure of human consciousness. Aust N Z J Psychiatry 43(11): 9941006.

26. Defelipe J (2011) The evolution of the brain, the human nature of cortical circuits, and intellectual creativity.Front Neuroanat. 5: 29.

27. Kaas JH (2013) The evolution of brains from early mammals to humans. Wiley Interdiscip Rev Cogn Sci 4(1): 33-45.

28. Karten HJ (2015) Vertebrate brains and evolutionary connectomics: on the origins of the mammalian 'neocortex'. Philos Trans R Soc Lond B Biol Sci 370(1684): pii: 20150060.

29. Naumann RK, Ondracek JM, Reiter S, Shein-Idelson M, Tosches MA, et al. (2015) The reptilian brain. Curr Biol 25(8): R317-R321.

30. Wynn T, Coolidge FL (2016) Archeological insights into hominin cognitive evolution. Evol Anthropol 25(4): 200-213.

31. Averof M, Cohen SM (1997) Evolutionary origin of insect wings from ancestral gills. Nature 385(6617): 627-630

32. Pearson BJ, Sánchez Alvarado A (2008) Regeneration, stem cells, and the evolution of tumor suppression. Cold Spring Harb Symp Quant Biol 73: 565-572.

33. Nam H, Conrad TM, Lewis NE (2011) The role of cellular objectives and selective pressures in metabolic pathway evolution. Curr Opin Biotechnol 22(4): 595-600. 